\title{
Plastic response of conical shells with stiffeners to blast loading
}

\author{
JaAn Lellep and Ella Puman
}

\begin{abstract}
The inelastic response of circular conical shells to the blast loading is studied. The impact loading is applied at the initial time moment and it is removed at a certain instant of time. The load intensity depends of the coordinate of the shell. The material of the shell is a perfect plastic one obeying the Johansen yield condition and the associated flow law. It is assumed that the frustum of the cone is furnished with ring stiffeners made of the same material. A theoretical method for the evaluation of the stress strain state of the shell and for determination of maximal residual deflections is developed.
\end{abstract}

\section{Introduction}

The blast loading of structural elements occurs in real life situations as traffic accidents, ship collision, metal forming and so on. The dynamic plastic response of axisymmetric plates is investigated by Jones [1], [2], Shen and Jones [7], Wang et al. [8]. In the literature much less attention has been paid to conical shells. Lellep and Puman [3], [4], [5] studied the plastic response of conical shells to the rectangular blast loading, where loading intensity decays according to the exponential law. In the present paper, a theoretical method is developed for the shell, loaded by the pressure uniformly distributed over the shell. It is assumed that the load intensity decreases from inner edge to the outer edge.

\section{Problem formulation}

Consider the response of a conical shell to the distributed dynamic loading. Let the shell be absolutely free at the inner edge $r=a$ and be simply

Received November 1, 2018.

2010 Mathematics Subject Classification. 74C05.

Key words and phrases. Blast loading, plasticity, conical shell, stiffener, stepped thickness.

https://doi.org/10.12697/ACUTM.2020.24.01 
supported at the outer edge $r=R$ (Figure 1), where $r$ stands for the current radius of the shell. Let the intensity of the uniformly distributed transverse loading be $P(t, r)$ where

$$
P(t, r)=\left\{\begin{array}{lc}
P_{*} \frac{r-R}{a-R}, & t \in\left[0, t_{1}\right], \\
0, & t>t_{1} .
\end{array}\right.
$$

Here $P_{*}$ is a given constant and $t_{1}$ is the time moment, when loading will be removed.

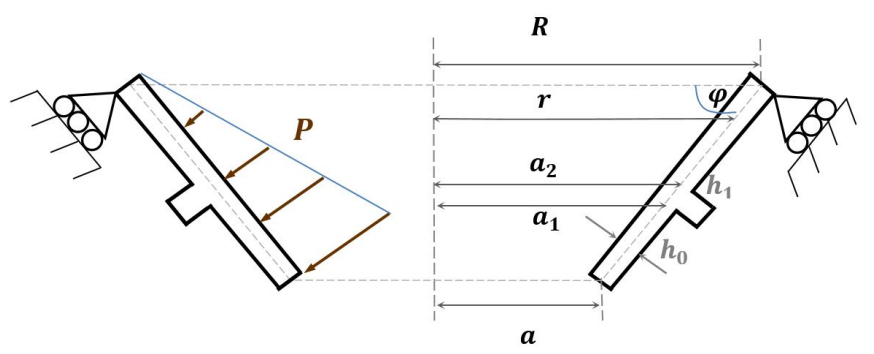

Figure 1. Conical shell with a stiffener.

In the present study the shell with the stiffeners is modelled as the shell of stepped thickness. It is expected that this simplification does not proceed large discrepancies of results in comparison to those corresponding to the exact model of the shell. Let the thickness of the shell wall be $h$, where

$$
h= \begin{cases}h_{0}, & r \in\left[a, a_{1}\right], \\ h_{1}, & r \in\left[a_{1}, a_{2}\right], \\ h_{0}, & r \in\left[a_{2}, R\right] .\end{cases}
$$

The equilibrium equations for the conical shell element are

$$
\left(r N_{1}\right)^{\prime}-N_{2}=0
$$

and

$$
\left(\left(r M_{1}\right)^{\prime}-M_{2}\right)^{\prime}-N_{2} \frac{\sin \varphi}{\cos ^{2} \varphi}+\frac{r}{\cos ^{2} \varphi}\left(P_{*} \frac{r-R}{a-R}-h \bar{\mu} \ddot{W}\right)=0,
$$

where prime denotes the differentiation with respect to current radius $r$. In (2) and (3) $N_{1}$ and $N_{2}$ are membrane forces, $M_{1}$ and $M_{2}$ are bending moments in the radial and circumferential directions, and $\varphi$ stands for the angle of inclination of a generator of the middle surface of the shell (Figure 1). The displacements $U$ and $V$ in the radial and circumferential directions are 
assumed to be small in comparison to the transverse deflection $W$. The strain rate components can be presented as

$$
\begin{aligned}
& \dot{\varepsilon}_{1}=\frac{d \dot{U}}{d r} \cos \varphi, \\
& \dot{\varepsilon}_{2}=\frac{1}{r}(\dot{U} \cos \varphi+\dot{W} \sin \varphi),
\end{aligned}
$$

and

$$
\begin{aligned}
& \dot{\kappa}_{1}=-\frac{d^{2} \dot{W}}{d r^{2}} \cos ^{2} \varphi \\
& \dot{\kappa}_{2}=-\frac{1}{r} \frac{d \dot{W}}{d r} \cos ^{2} \varphi,
\end{aligned}
$$

where $\varepsilon_{1}, \varepsilon_{2}$ are linear elongations and $\kappa_{1}, \kappa_{2}$ are curvatures. Let us introduce the following non-dimensional quantities

$$
\begin{aligned}
\varrho & =\frac{r}{R}, \quad \alpha_{l}=\frac{a_{l}}{R}, \quad l=0,1,2, \gamma_{j}=\frac{h_{j}}{h_{*}}, j=0,1, \\
n_{i} & =\frac{N_{i}}{N_{*}}, m_{i}=\frac{M_{i}}{M_{*}}, \quad i=1,2, \\
p_{*} & =\frac{P_{*} R}{N_{*} \sin \varphi}, \quad \mu=\frac{\bar{\mu} R h_{*}}{N_{*} \sin \varphi}, \quad k=\frac{M_{*} \cos \varphi}{R N_{*} \sin \varphi} .
\end{aligned}
$$

Here $h_{*}$ stands for the thickness of the reference shell, $M_{*}=\frac{\sigma_{0} h_{*}^{2}}{4}$, and $N_{*}=\sigma_{0} h_{*}$ are the yield moment and the yield force, and $\sigma_{0}$ is the yield stress of the material.

\section{The stress strain state of the shell}

The stress profile could lie on sides $A_{1} B_{1}$ and $A B$ of the yield loci (Figure 2). From these regimes and from the associated flow law it follows that

$$
\dot{\varepsilon}_{1}=0, \dot{\kappa}_{1}=0 .
$$

Thus from (4) and (5) one obtains

$$
\dot{U}^{\prime}=0, \dot{W}^{\prime \prime}=0 .
$$

After integration with respect to $\varrho$ one has

$$
\dot{W}=C_{1}(t) \varrho+C_{2}(t),
$$

where $C_{1}(t)$ and $C_{2}(t)$ are arbitrary functions. From the boundary requirements

$$
\dot{U}(1, t)=0, \dot{W}(\alpha, t)=\dot{w}_{0}, \dot{W}(1, t)=0,
$$

were $w_{0}$ is unknown function, we can deduce that

$$
\dot{U}=\text { const, } \dot{W}=\dot{w}_{0} \frac{\varrho-1}{\alpha-1}, \ddot{W}=\ddot{w}_{0} \frac{\varrho-1}{\alpha-1} .
$$




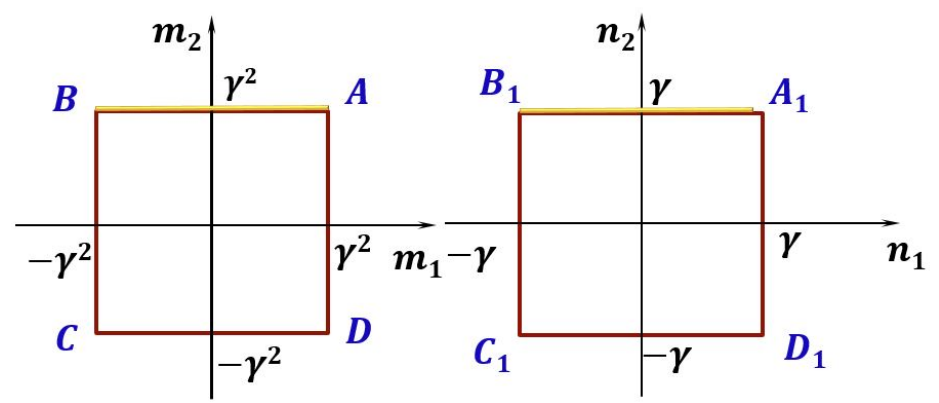

Figure 2. Johansen's yield condition.

\section{Equations of motion}

The equations of motion in non-dimensional variables are

$$
\left(\varrho n_{1}\right)^{\prime}-n_{2}=0
$$

and

$$
k\left(\left(\varrho m_{1}\right)^{\prime}-m_{2}\right)^{\prime}-n_{2}+\varrho\left(p_{*} \frac{\varrho-1}{\alpha-1}-\gamma \mu \ddot{W}\right)=0,
$$

where $p_{*}$ is given constant. Making use of (6), one can rewrite the equation (8) as

$$
k\left(\left(\varrho m_{1}\right)^{\prime}-m_{2}\right)^{\prime}-n_{2}+\varrho \frac{\varrho-1}{\alpha-1}\left(p_{*}-\gamma \mu \ddot{w}_{0}\right)=0 .
$$

The boundary conditions for free inner edge and simply supported outer edge are

$$
n_{1}(\alpha, t)=0, q(\alpha, t)=0, m_{1}(\alpha, t)=0, m_{1}(1, t)=0,
$$

where $q=\left(\varrho m_{1}\right)^{\prime}-m_{2}$ is non-dimensional shear force. After integration of the equations (7) and (9) with respect to $\varrho$ and finding the constants of integration, one obtains

$$
\begin{aligned}
n_{1}= & \gamma\left(1-\frac{\alpha}{\varrho}\right), \\
m_{1}= & \gamma^{2}\left(1-\frac{\alpha}{\varrho}\right)+\frac{\gamma}{k}\left(\frac{\gamma}{2}-\alpha+\frac{\alpha^{2}}{2 \gamma}\right) \\
& +\frac{p_{*}-\mu \gamma \ddot{w}_{0}}{12 k(\alpha-1)}\left(\varrho^{2}(2-\varrho)+2 \alpha^{2}(2 \alpha-3)-\frac{\alpha^{3}}{\varrho}(3 \alpha-4)\right) .
\end{aligned}
$$

The acceleration at the shell center $\ddot{w}_{0}$ in (11) can be obtained by using the last boundary condition in (10):

$$
\mu \ddot{w}_{0}=\frac{p_{*}}{\gamma}+\frac{12 k(\alpha-1)}{(3 \alpha+1)(1-\alpha)^{3}}\left(\gamma(1-\alpha)+\frac{(1-\alpha)^{2}}{2 k}\right) .
$$


At the time period $t>t_{1}$, the acceleration is notated by $\ddot{w}_{1}=\ddot{w}(\alpha, t)$, then $p_{*}=0$ and

$$
\mu \ddot{w}_{1}=\frac{12 k(\alpha-1)}{(3 \alpha+1)(1-\alpha)^{3}}\left(\gamma(1-\alpha)+\frac{(1-\alpha)^{2}}{2 k}\right) .
$$

After integration of (12) with respect to the time $t$, one obtains

$$
\begin{aligned}
& \mu \dot{w}_{0}=\frac{p_{*}}{\gamma} t+\frac{12 k(\alpha-1) t}{(3 \alpha+1)(1-\alpha)^{3}}\left(\gamma(1-\alpha)+\frac{(1-\alpha)^{2}}{2 k}\right), \\
& \mu w_{0}=\frac{p_{*} t^{2}}{2 \gamma}+\frac{6 k(\alpha-1) t^{2}}{(3 \alpha+1)(1-\alpha)^{3}}\left(\gamma(1-\alpha)+\frac{(1-\alpha)^{2}}{2 k}\right) .
\end{aligned}
$$

The peak value of the linearly distributed static plastic collapse pressure $p_{0}$ can be evaluated from (12), when $\ddot{w}_{0}=0$ :

$$
p_{0}=\frac{6 \gamma(2 k \gamma+1-\alpha)}{(1-\alpha)(1+3 \alpha)} \text {. }
$$

The shell thickness (1) in non-dimensional variables for the shell with a single stiffener is

$$
\gamma= \begin{cases}\gamma_{0}, & \varrho \in\left[\alpha, \alpha_{1}\right], \\ \gamma_{1}, & \varrho \in\left[\alpha_{1}, \alpha_{2}\right], \\ \gamma_{0}, & \varrho \in\left[\alpha_{2}, 1\right] .\end{cases}
$$

According to the Johansen's yield condition, one can assume that

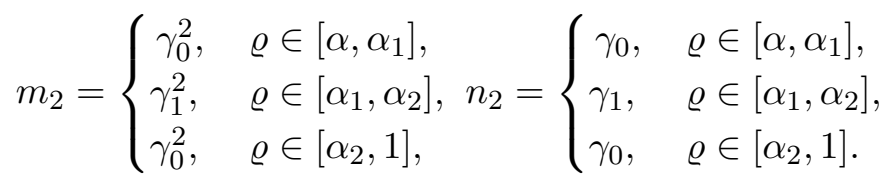

After integration of the equations of motion in three different regions, the radial membrane force obtains:

$$
n_{1}= \begin{cases}\gamma_{0}-\frac{\gamma_{0} \alpha}{\varrho}, & \varrho \in\left[\alpha, \alpha_{1}\right], \\ \gamma_{1}+\frac{1}{\varrho}\left(\alpha_{1}\left(\gamma_{0}-\gamma_{1}\right)-\gamma_{0} \alpha\right), & \varrho \in\left[\alpha_{1}, \alpha_{2}\right], \\ \gamma_{0}+\frac{1}{\varrho}\left(\alpha_{2}\left(\gamma_{1}-\gamma_{0}\right)+\alpha_{1}\left(\gamma_{0}-\gamma_{1}\right)-\gamma_{0} \alpha\right), & \varrho \in\left[\alpha_{2}, 1\right] .\end{cases}
$$

Similarily, one obtains the radial bending moment for $\varrho \in\left[\alpha, \alpha_{1}\right]$ :

$$
m_{1}=\gamma_{0}^{2}+\frac{\gamma_{0}}{k}\left(\frac{\varrho}{2}-\alpha\right)-\frac{p_{*}-\mu \gamma_{0} \ddot{w}_{0}}{12 k(\alpha-1)} \varrho^{2}(\varrho-2)+\frac{C_{11}}{k}+\frac{C_{21}}{k \varrho},
$$

for $\varrho \in\left[\alpha_{1}, \alpha_{2}\right]$ :

$m_{1}=\gamma_{1}^{2}+\frac{\gamma_{1}}{k}\left(\frac{\varrho}{2}-\alpha_{1}\right)+\frac{\gamma_{0}}{k}\left(\alpha_{1}-\alpha\right)-\frac{p_{*}-\mu \gamma_{1} \ddot{w}_{0}}{12 k(\alpha-1)} \varrho^{2}(\varrho-2)+\frac{C_{12}}{k}+\frac{C_{22}}{k \varrho}$, 
and for $\varrho \in\left[\alpha_{2}, 1\right]$ :

$$
\begin{aligned}
m_{1}= & \gamma_{0}^{2}+\frac{\gamma_{0}}{k}\left(\frac{\varrho}{2}+\alpha_{1}-\alpha-\alpha_{2}\right)+\frac{\gamma_{1}}{k}\left(\alpha_{2}-\alpha_{1}\right) \\
& -\frac{p_{*}-\mu \gamma_{0} \ddot{w}_{0}}{12 k(\alpha-1)} \varrho^{2}(\varrho-2)+\frac{C_{13}}{k}+\frac{C_{23}}{k \varrho} .
\end{aligned}
$$

The constants of integration are

$$
\begin{aligned}
C_{11}= & \frac{p_{*}-\mu \gamma_{0} \ddot{w}_{0}}{6(\alpha-1)}\left(2 \alpha^{3}-3 \alpha^{2}\right), \\
C_{21}= & -k \alpha \gamma_{0}^{2}+\gamma_{0} \frac{\alpha^{2}}{2}+\frac{p_{*}-\mu \gamma_{0} \ddot{w}_{0}}{12(\alpha-1)}\left(\alpha^{4}-2 \alpha^{3}\right)-C_{11} \alpha, \\
C_{12}= & \frac{\mu \ddot{w}_{0}\left(\gamma_{0}-\gamma_{1}\right)}{6(\alpha-1)}\left(2 \alpha_{1}^{3}-3 \alpha_{1}^{2}\right)+C_{11}, \\
C_{22}= & k \alpha_{1}\left(\gamma_{0}^{2}-\gamma_{1}^{2}\right)+\frac{\alpha_{1}^{2}}{2}\left(\gamma_{1}-\gamma_{0}\right) \\
& +\frac{\mu \ddot{w}_{0}}{12(\alpha-1)}\left(\gamma_{0}-\gamma_{1}\right)\left(\alpha_{1}^{4}-2 \alpha_{1}^{3}\right)+\alpha_{1}\left(C_{11}-C_{12}\right)+C_{21}, \\
C_{13}= & \frac{\mu \ddot{w}_{0}\left(\gamma_{1}-\gamma_{0}\right)}{6(\alpha-1)}\left(2 \alpha_{2}^{3}-3 \alpha_{2}^{2}\right)+C_{12}, \\
C_{23}= & k \alpha_{2}\left(\gamma_{1}^{2}-\gamma_{0}^{2}\right)+\frac{\alpha_{2}^{2}}{2}\left(\gamma_{0}-\gamma_{1}\right) \\
& +\frac{\mu \ddot{w}_{0}}{12(\alpha-1)}\left(\gamma_{1}-\gamma_{0}\right)\left(\alpha_{2}^{4}-2 \alpha_{2}^{3}\right)++\alpha_{2}\left(C_{12}-C_{13}\right)+C_{22} .
\end{aligned}
$$

The acceleration for the shell with a stiffener at the internal edge of the shell $\ddot{w}_{0}$ can be determined using the last boundary condition of (10) as

$$
\mu \ddot{w}_{0}=\frac{12 k(\alpha-1)}{N} L,
$$

where

$$
\begin{aligned}
N= & \gamma_{0}(1+3 \alpha)(1-\alpha)^{3}+\left(\gamma_{0}-\gamma_{1}\right)\left(\left(1+3 \alpha_{1}\right)\left(\alpha_{1}-1\right)^{3}+\left(1+3 \alpha_{2}\right)\left(1-\alpha_{2}\right)^{3}\right), \\
L= & \frac{p_{*}}{12 k(\alpha-1)}(1+3 \alpha)(1-\alpha)^{3}+\gamma_{0}^{2}\left(1-\alpha+\alpha_{1}-\alpha_{2}\right) \\
& +\gamma_{1}^{2}\left(\alpha_{2}-\alpha_{1}\right)+\frac{\gamma_{0}}{2 k}(1-\alpha)^{2}+\frac{1}{2 k}\left(\gamma_{0}-\gamma_{1}\right)\left(\alpha_{2}^{2}-\alpha_{1}^{2}+2\left(\alpha_{1}-\alpha_{2}\right)\right) .
\end{aligned}
$$

\section{Numerical results}

The results of calculations are presented in Figures 3-13. In Figure 3 values of the lower limits of the pressure $p_{0}$, corresponding to the onset of plastic deformations, are shown for shell with constant thickness for different inner radii. The lowest curve is obtained for the value of the parameter 
$k=0.1$ and the highest curve for $k=0.9$. It can be seen from Figure 3 that if the angle of inclination of the conical shell increases, then the lower limit of the pressure increases as well.

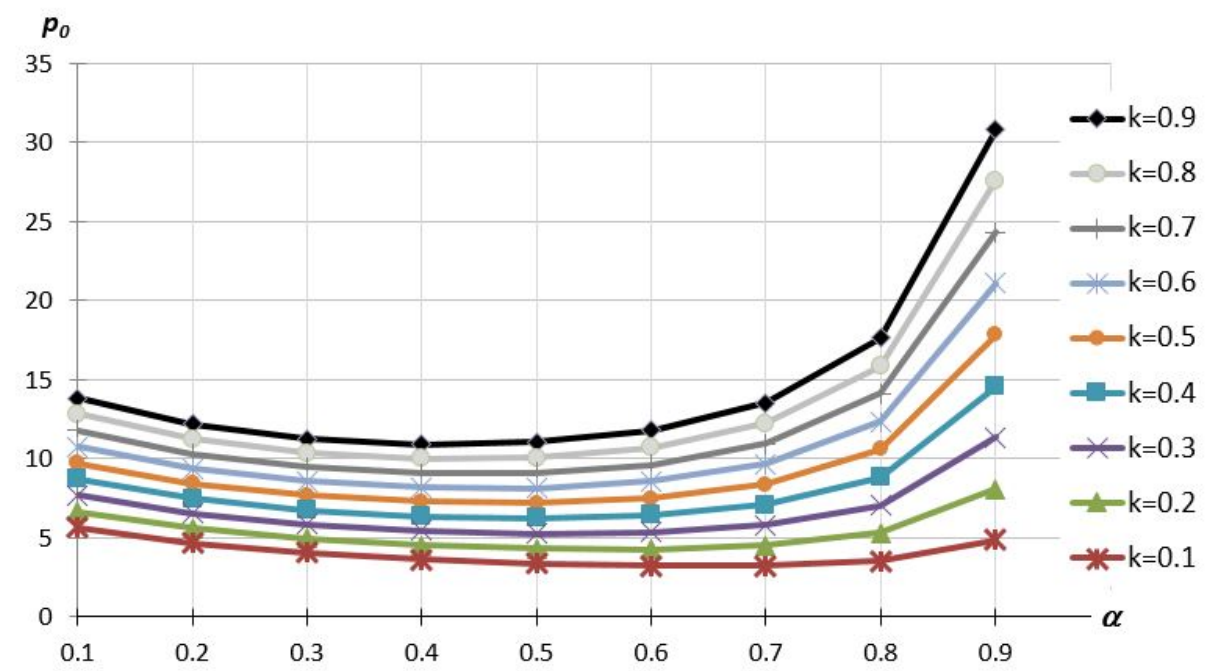

FIgURE 3. Lower limit of the pressure for different inner radii.

The volume of the shell material for the shell with stiffener is

$$
V_{s}=\gamma_{0}\left(\alpha_{1}-\alpha\right)+\gamma_{1}\left(\alpha_{2}-\alpha_{1}\right)+\gamma_{0}\left(1-\alpha_{2}\right),
$$

Similarily, for the shell with constant thickness $\gamma$, the volume is

$$
V_{c}=\gamma(1-\alpha) .
$$

Then from $V_{s}=V_{c}$ we get the value of the constant thickness

$$
\gamma=\frac{\gamma_{0}\left(\alpha_{1}-\alpha\right)+\gamma_{1}\left(\alpha_{2}-\alpha_{1}\right)+\gamma_{0}\left(1-\alpha_{2}\right)}{1-\alpha} .
$$

The maximal residual deflection for different heights of the stiffener are depicted in Figure 4. The dash lines represent the shells with constant thicknesses and solid lines the shells with a stiffener. The upper dashed line represents the maximal residual deflections for the shell with constant thickness $\gamma=1.075$, which has the same material volume as the shell with a single stiffener with thickness $\gamma_{1}=1.5$. The lower dashed line represents the shell with constant thickness $\gamma=1.103$ with the same material volume as the shell with stiffener, when the stiffener thickness is $\gamma_{1}=2$. It can be seen from Figure 4 that if the stiffener thickness increases, the maximal residual deflection decreases, as might be expected. For the same material 


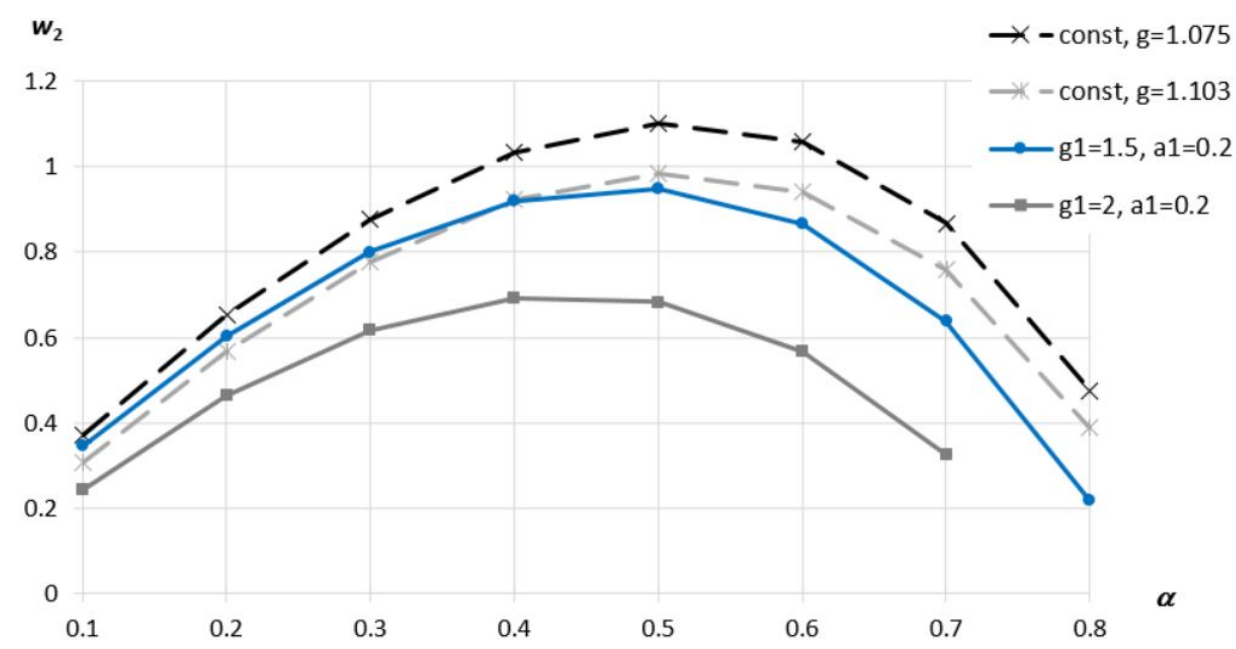

Figure 4. Maximal residual deflections for shells with constant thickness and for shells with a stiffener.

volume, one can achieve lower maximal residual deflections when using stiffeners. In Figure 5 the maximal residual deflections are depicted for different stiffener locations for $\alpha=0.3,0.5,0.6$ and 0.7 . It can be seen from Figure 5 that stiffener location near the inner edge provides lower maximal residual deflections.

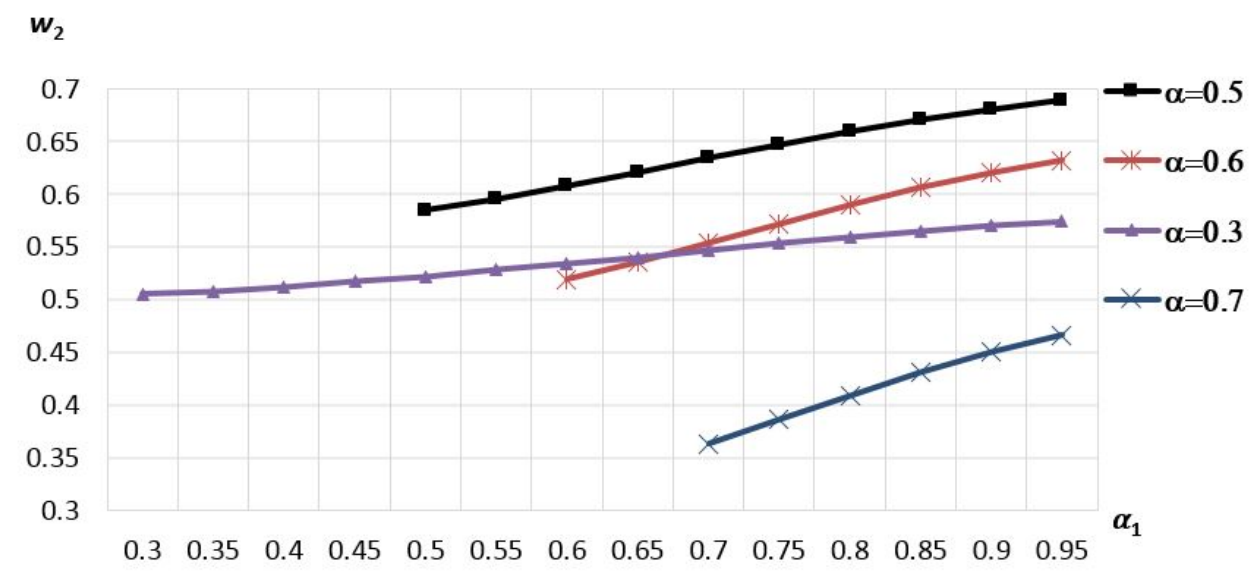

FiguRE 5. Maximal residual deflections for different stiffener locations.

The maximal residual deflections for load intensity $p_{*}=0.5$ and $k=0.3$ are presented in Figure 6 for different loading times $t_{1}$ and inner radii. Here 
$\alpha_{1}=\alpha+0.1, \alpha_{2}=\alpha_{1}+0.05, \gamma_{0}=1.05, \gamma_{1}=1.5$. It can be seen from Figure 6 that longer loading time causes larger maximal residual deflections.

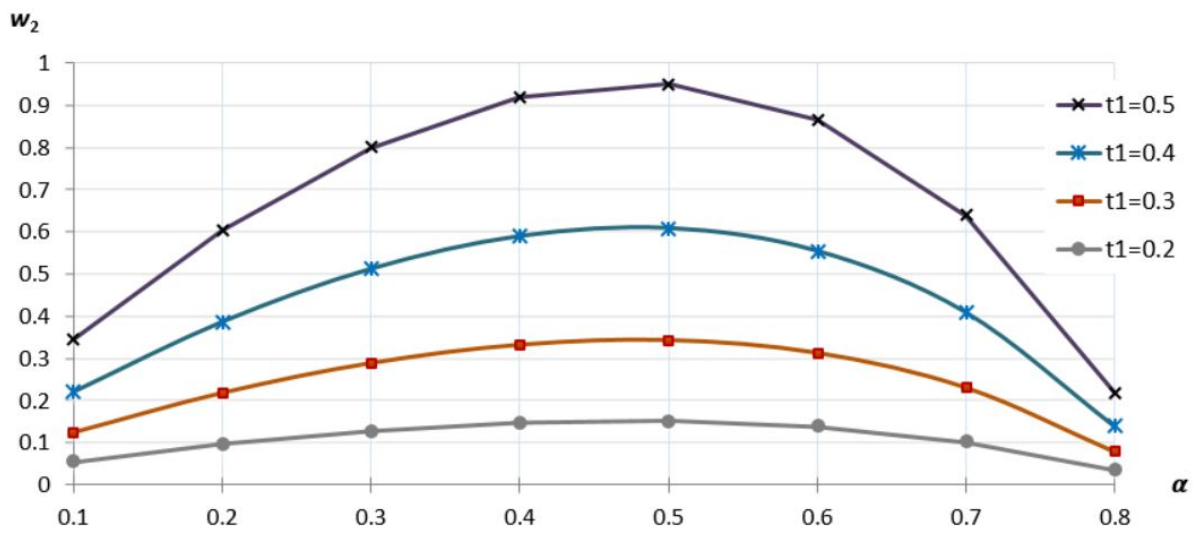

FiguRE 6. Maximal residual deflections for different inner radii and loading times.

The maximal residual deflections for loading time $t_{1}=0.5$ and $k=0.3$ are presented in Figure 7 and Table 1 for different inner radii and load intensities. The residual deflection attains its maximum if the inner radius $\alpha$ is about 0.5 for larger load intensity values.

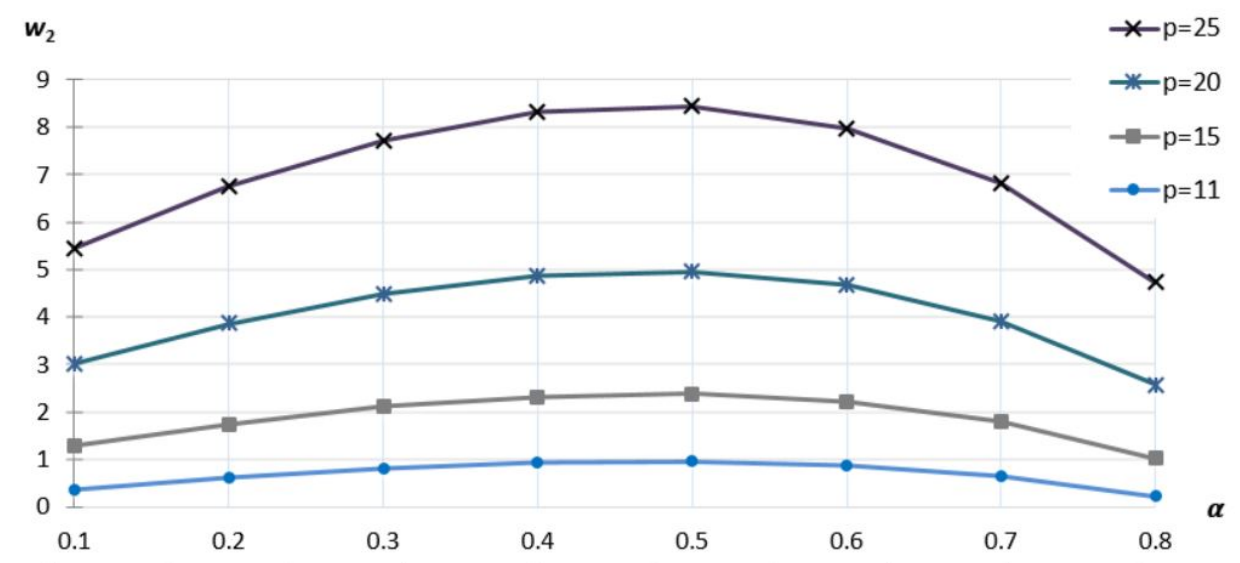

Figure 7. Maximal residual deflections for different inner radii and load intensities. 
The maximal residual deflections for different values of the parameter $k$ are presented in Figure 8. If the value of the parameter $k$ decreases, then the angle of inclination of the middle surface of the shell increases and the maximal residual deflections have greater values. It can be seen from Figure 8 that, in the case of small values of $k$, the residual deflections increase together with the inner radius $\alpha$. However, in the case of moderate values of $k(k>0.01)$, maximal deflections decrease with increasing the internal radius of the shell if $a>R / 2$. The result coincides with our expectations as, evidently, a narrow annulus deforms less than an annular plate with a comparatively small central hole.

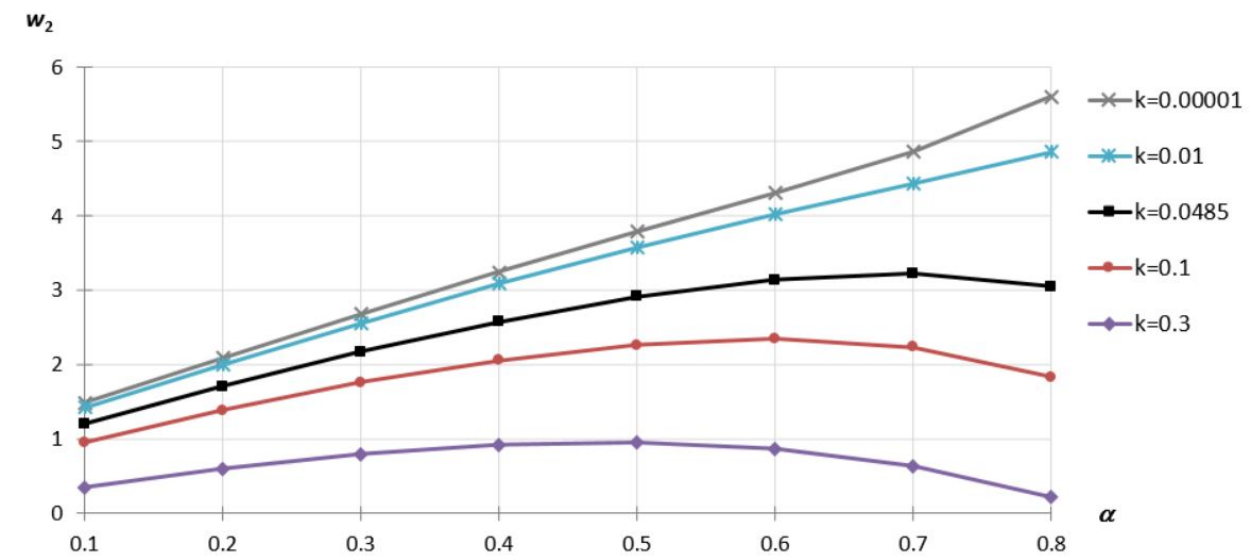

FiguRE 8. Maximal residual deflections for different angle of inclination and inner radii.

The radial bending moment distributions are presented in Figures 9-11 for $\alpha=0.1$ and $\alpha=0.5$ for different stiffener locations $\alpha_{1}$ and different stiffener thicknesses $\gamma_{1}=1.5$ and $\gamma_{1}=2$. The dashed lines in Figures 9 11 represent the shells with constant thickness so that the volumes of the material of the shell with constant thickness and the shell with stiffener are equal. From Figures 9-11 we can see that the stiffener location and stiffener thickness cause the changes of values of the bending moment.

In Figures 9-11 the distributions of the radial bending moment are presented for the shell with the internal radius $a=R / 2$.

In Figure 11 the stiffener thicknesses are $\gamma_{1}=1.5$ and $\gamma_{1}=2$ for the inner radii $\alpha=0.5$. Stiffeners are located in the intervals $[0.5,0.55],[0.6,0.65]$ and $[0.7,0.75]$ for both cases.

The results of calculations are presented for different loading times and inner radii for $k=0.3, p_{*}=11, \gamma_{0}=1.05, \gamma_{1}=1.5, \alpha_{2}=\alpha_{1}+0.05 \mathrm{in}$ Table 2. The stiffener locations are near the inner radius with the step 0.1. 


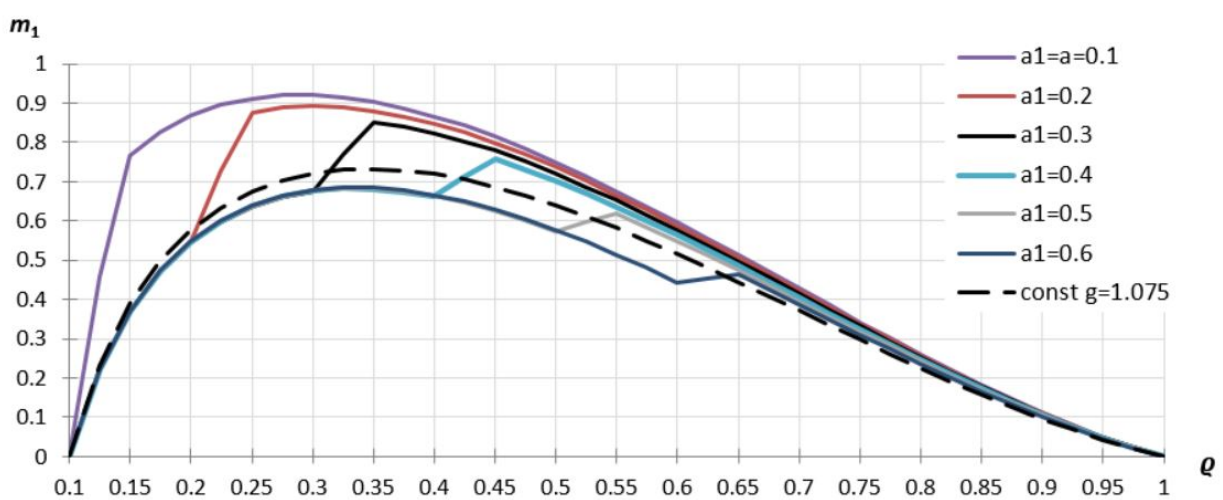

FIgURE 9. Sensitivity of the radial bending moment on the stiffener location.

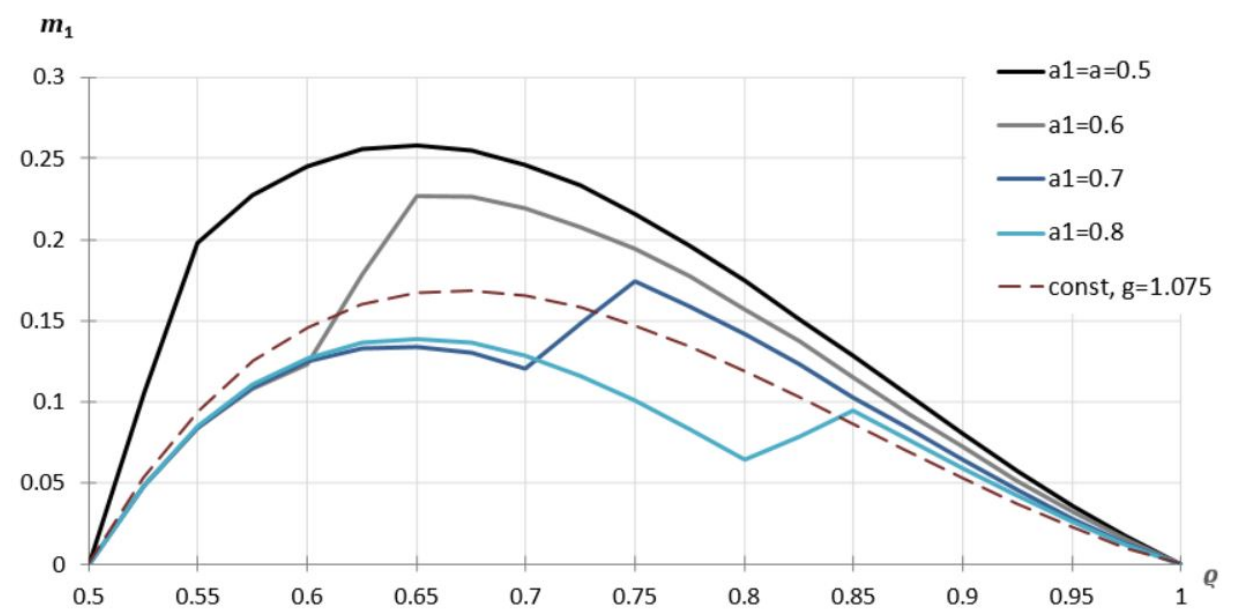

Figure 10. Sensitivity of the radial bending moment on the stiffener location.

From Table 2 we can see that the maximal residual deflection values are higher for longer loading time and for inner radius $\alpha=0.5$, as might be expected. The total response time $t_{2}$ increases together with $t_{1}$.

The results of the current paper regarding the quasi static and impact loading are compared with corresponding results by Sawczuk and SokolSupel [6] and Jones [2] in Figures 12, 13. In Figure 12 the limit loads for the conical shell are compared with the limit load of the annular plate with the same external and internal radii. The lower curve corresponds to the conical frusta with the angle of inclination $\varphi=0.034^{\circ}$, the corresponding value of the parameter $k$ is $k=100$, if $h=0.24 R$. It can be seen from Figure 12 


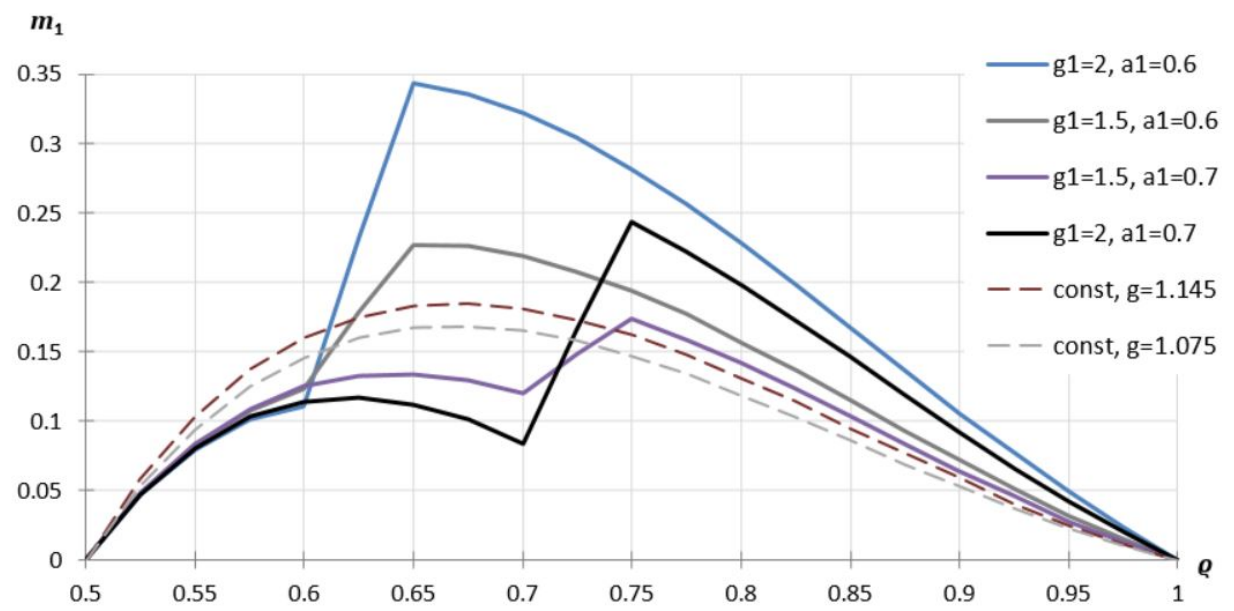

FiguRE 11. Radial bending moment distributions for $\alpha=$ 0.5 and for different stiffener thicknesses and locations.

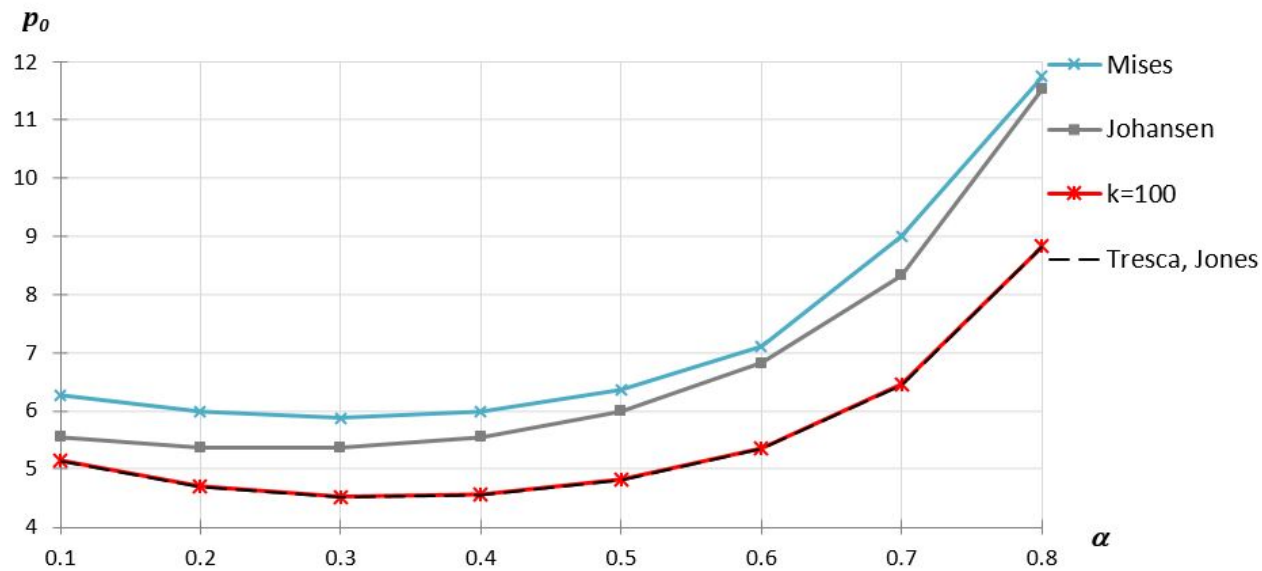

FiguRE 12. Limit loads for conical shells compared with annular plates for different yield conditions.

that the limit load for the conical shell tends to the value corresponding to the load carrying capacity of the annular plate, as might be expected.

In Figure 13 a similar comparison has been undertaken for the conical shell, respectively, subjected to the dynamic pressure. Here the transverse load intensity is varying linearly from the value $p_{0}$ at the internal edge until zero at the external edge of the shell. The results obtained in the present paper for conical frusta coincide in the limit case $\varphi=0$ with those obtained by Jones [2]. The solution of Jones is presented in Figure 13 by the dashed 


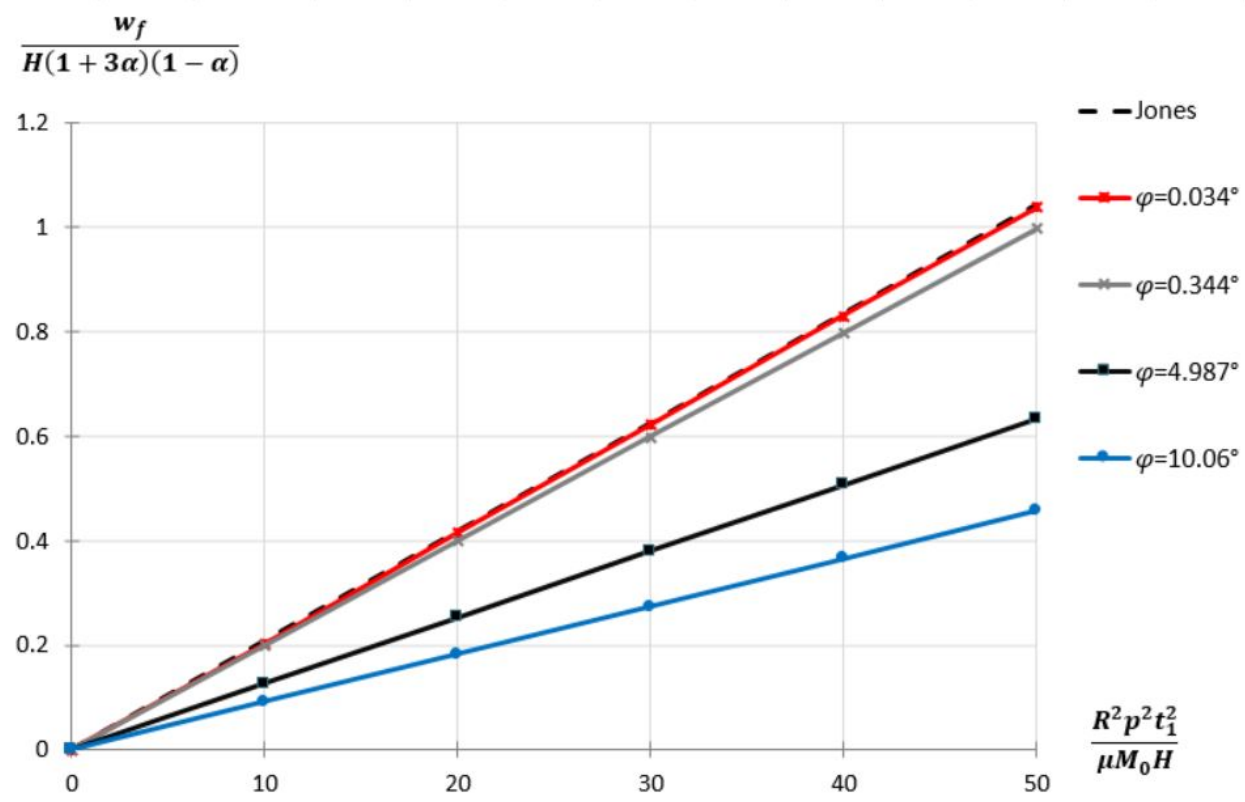

Figure 13. Maximal residual deflection for annular plate compared with conical shell for different angles of inclination.

line. Here the curves correspond to the angles of inclination of the conical shell from lower line with $\varphi=10^{\circ}, 5^{\circ}, 0.344^{\circ}, 0.034^{\circ}$ (corresponding values of the parameter $k$ are $k=0.333,0.685,10,100$, in the case $h=0.24 R$, $\left.p_{*}=2 p_{0}\right)$.

\section{Concluding remarks}

A theoretical method for the determination of maximal residual deflections of circular conical shells with a stiffener was developed. It was shown that when using a stiffener, one can reduce the maximal residual deflection. It appeared that the best position for the stiffener is somewhere near the free inner edge. This regards to the case of small deflections. The case of large deflections needs additional investigation.

\section{Acknowledgement}

This work was supported by the Institutional Research Funding IUT20-57 of the Estonian Ministry of Education and Research.

\section{References}

[1] N. Jones, The collapse pressure of a flush cylindrical nozzle intersecting a conical pressure vessel axisymmetrically, Int. J. Mech. Sci. 11 (1969), 401-415. 
[2] N. Jones, Structural Impact, CUP: Cambridge, 2012.

[3] J. Lellep and E. Puman, Plastic behaviour of stepped conical shells under impact loading, WIT Transactions on the Built Environment 126 (2012), 15-25.

[4] J. Lellep and E. Puman, Blast loading of inelastic conical shells with stiffeners, in: Recent Advances in Mechanical Engineering, 2014, pp. 77-83.

[5] J. Lellep and E. Puman, Response of stepped conical shells to the blast loading, Int. J. Mech. 10 (2016), 167-174.

[6] A. Sawczuk and J. Sokol-Supel, Limit analysis of plates, PWN, Warszawa, 1993.

[7] W. Q. Shen and N. Jones, Dynamic response and failure of fully clamped circular plates under impulse loading, Int. J. Impact Eng. 13 (1993), 259-278.

[8] Y. Wang, M. Yu, Y. Xiao, and L. Li, Dynamic plastic response of a circular plate based on unified strength theory, Int. J. Impact Eng. 31 (2005), 25-40.

Institute of Mathematics and Statistics, University of TARtu, 18 Narva MNT., 51009, TARTU, Estonia

E-mail address: jaan.lellep@ut.ee

E-mail address: ella.puman@ut.ee 